\title{
20. NEOGENE BENTHIC FORAMINIFERS FROM DSDP SITES 360 AND 362, SOUTH EASTERN ATLANTIC
}

\author{
Alexa Cameron, Department of Geology, University of Canterbury, Christchurch, New Zealand
}

Miocene to Quaternary benthic foraminifers have been examined from the South Atlantic Sites 360 and 362 (Figure 1). Site 360 is situated midway down the continental rise off Southwest Africa, at latitude $35^{\circ} 51^{\prime} \mathrm{S}$, longitude $18^{\circ} 05^{\prime} \mathrm{E}$, in a water depth of 2977 meters. Site 362 is situated on the Abutment Plateau of the Frio Ridge segment of the Walvis Ridge, at latitude $19^{\circ} 45^{\prime} \mathrm{S}$, longitude $10^{\circ} 32^{\prime} \mathrm{E}$, in a water depth of 1325 meters.

The portions examined are: Site 360, Cores 1-25 (lower Pliocene-lower Miocene); Site 362, Cores 1-38 (Pleistocene-lower Miocene).

Most of the material examined consists of foraminifer- or diatom-rich nannofossil ooze and chalks. The benthic foraminifers are sparse, and in many instances only 1-2 specimens were found for each species present (Tables 1 and 2).

The benthics appear as a very small portion of the total foraminifer fauna, in most cases less than $1 \%$ of the sample provided.

The benthic faunas are dominated by long-ranging, moderate to deep-water species, not particularly suitable for biostratigraphic subdivision. Miliolids, mainly Quinqueloculina, and Biloculina are persistent throughout Sites 360 and 362 .

Arenaceous forms were found with six species present at Site 360, and nine at Site 362. From the range charts (Tables 1 and 2) it can be seen that the dominant and persistent forms are: Pullenia bulloides, Pullenia quinqueloba, Oridorsalis umbonatus, Oridorsalis tenera, Cassidulina subglobosa, Cassidulina laevigata. Alabumina tenuimarginata, Sphaeroidina bulloides, Martinottiella communis, Cibicides ihungia, Cibicides molestus, Bulimina truncanella, Angulogerina esuriens, Gyroidina prominula and Gyroidinoides zelandicus. Less common, though still present, are: Hopkinsina mioindex, Euuvigerina notohispida, Bolivina finlayi, Laticarinina halophora and various species of Stilostomella.

The following species observed are known from the New Zealand sequence: Cibicides molestus, Cibicides ihungia, Bulimina marginata, Cassidulina laevigata, and Pullenia bulloides. Vella (1962a, b, c, 1963) inferred the following depth ranges for Miocene and Pliocene forms from the east coast of the North Island, New Zealand:

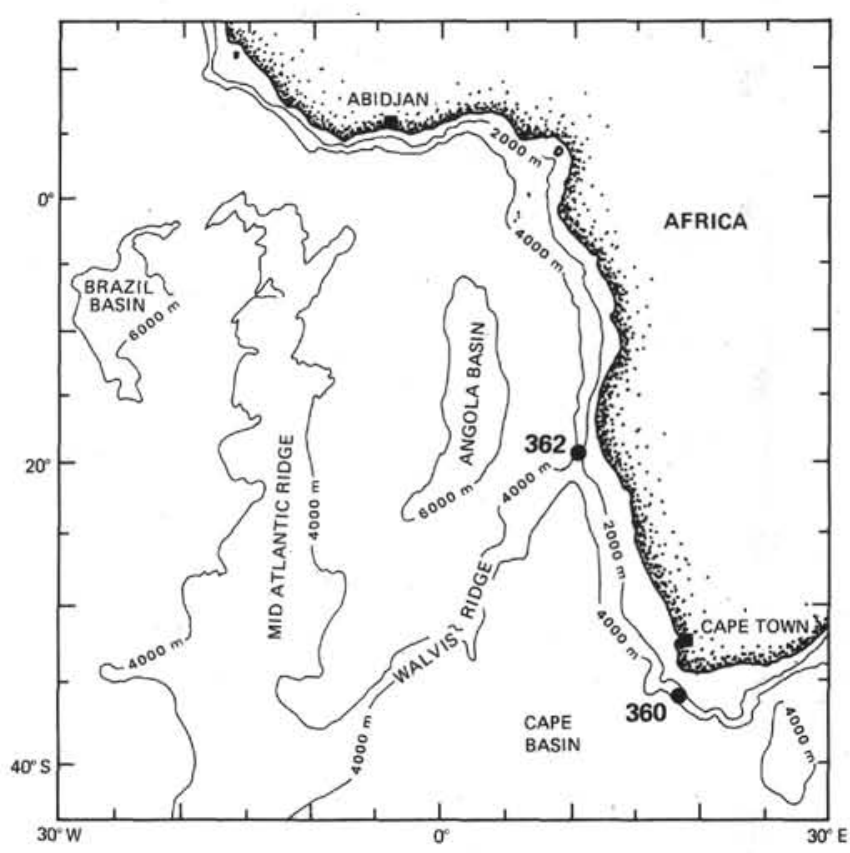

Figure 1. Location of Sites 360 and 362.

$\begin{array}{lr}\text { Pullenia bulloides } & 1000-4000 \mathrm{feet} \\ \text { Pullenia quinqueloba } & 400-1000 \mathrm{feet} \\ \text { Cibicides molestus } & 200-2000 \mathrm{feet} \\ \text { Cibicides ihungia } & 20-4000 \mathrm{feet} \\ \text { Cassidulina laevigata } & 20-1000 \mathrm{feet} \\ \text { Bulimina marginata } & 20-1000 \mathrm{feet}\end{array}$

\section{REFERENCES}

Vella, P.P., 1962a. Biostratigraphy and paleoecology of Mauriceville District, New Zealand: Trans. Roy. Soc. New Zealand Geol., v. 1, p. 183-199.

, 1962b. Determining depths of New Zealand Tertiary seas: Tuatara, v. 10, p. 19-40.

, 1962c. Late Tertiary Nonionid foraminifera from Wairarapa, New Zealand: Trans. Roy. Soc. New Zealand Geol., v. 1, p. 285-296.

1963. Some foraminifera from the upper Miocene and Pliocene of Wairarapa, New Zealand: Trans. Roy. Soc. New Zealand Geol., v. 2, p. 1-14. 
TABLE 1

Distribution of Lower Pliocene to Lower Miocene Benthic Foraminifers in Site 360

\begin{tabular}{|c|c|c|c|c|c|c|c|c|c|c|c|c|c|c|c|c|c|c|c|c|c|c|c|c|c|c|c|c|c|c|c|c|c|c|c|c|c|}
\hline \multicolumn{7}{|c|}{$\begin{array}{l}\text { LEG } 40 \\
\text { SITE } 360\end{array}$} & \multirow{2}{*}{ 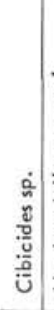 } & \multirow[b]{2}{*}{ 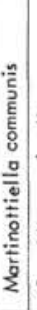 } & \multirow[b]{2}{*}{ 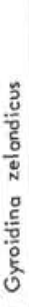 } & \multirow{2}{*}{ 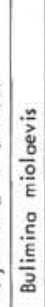 } & \multirow{2}{*}{ 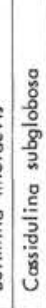 } & \multirow{2}{*}{ 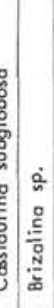 } & \multirow{2}{*}{ 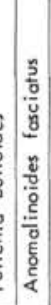 } & & & & & & & & & & $\frac{9}{2}$ & & & & & & & & & & & & & & \\
\hline 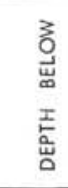 & & $\left\{\begin{array}{l}\tilde{w} \\
\frac{w}{\Sigma} \\
\underline{z}\end{array}\right.$ & & & 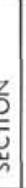 & $\begin{array}{l}\widehat{\underline{E}} \\
\vec{a} \\
\text { 竎 } \\
\underline{\underline{u}}\end{array}$ & & & & & & & & 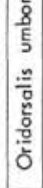 & 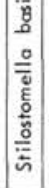 & 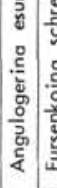 & 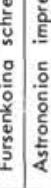 & 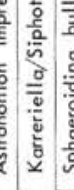 & 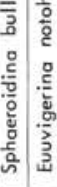 & 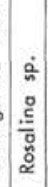 & 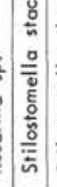 & 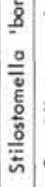 & 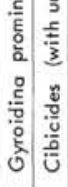 & 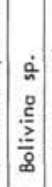 & 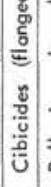 & 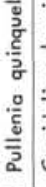 & 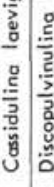 & $\begin{array}{l}\frac{2}{n} \\
0 \\
\frac{c}{\tilde{E}} \\
\frac{3}{3} \\
\frac{8}{8} \\
\frac{0}{0} \\
\end{array}$ & 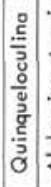 & $\begin{array}{l}\frac{z}{2} \\
\underline{y} \\
0 \\
\frac{5}{5} \\
\frac{0}{0} \\
\frac{8}{4} \\
\end{array}$ & 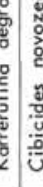 & 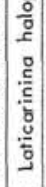 & 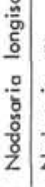 & & 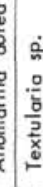 & 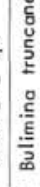 & $\overline{\overline{\hat{\theta}}}$ \\
\hline 79,5 & - & 89 & 1 & & 1 & $90-92$ & & & & & & & & & & & & & & & & & & & & & & & & & & & & & & & \\
\hline & & & 1 & & & CC & & & & & & & & & & & & & & & & & & & & & & & & & & & & & & & \\
\hline 89 & - & 98,5 & 2 & & 1 & $87-89$ & & & & & & & & & & & & & & & & & & & & & & & & & & & & & & & \\
\hline 98,5 & - & 108 & 3 & & 3 & $88-90$ & & & & & & & & & & & & & & & & & & & & & & & & & & & & & & & \\
\hline & & & 3 & & & $C C$ & & & & & & & & & & & & & & & & & & & & & & & & & & & & & & & \\
\hline 108 & - & 117,5 & 4 & & 3 & $89-91$ & & & & & & & & & & & & & & & & & & & & & & & & & & & & & & & \\
\hline 117,5 & - & 127 & 5 & & 3 & $107-109$ & & & & & & & & & & & & & & & & & & & & & & & & & & & & & & & \\
\hline & & & 5 & & & $C C$ & & & & & & & & & & & & & & & & & & & & & & & & & & & & & & & \\
\hline 127,5 & - & 136,5 & 6 & & 3 & $88-90$ & & & & & & & & & & & & & & & & & & & & & & & & & & & & & & & \\
\hline 136,5 & - & 146 & 7 & & 3 & $109-111$ & & & & & & & & & & & & & & & & & & & & & & & & & & & & & & & \\
\hline & & & 7 & & & CC & & & & & & & & & & & & & & & & & & & & & & & & & & & & & & & \\
\hline 146 & - & 155,5 & 8 & & 3 & $108-110$ & & & & & & & & & & & & & & & & & & & & & & & & & & & & & & & \\
\hline 155,5 & & 165 & s & & 3 & $86-88$ & & & & & & & & & & & & & & & & & & & & & & & & & & & & & & & \\
\hline & & & 9 & & & CC & & & & & & & & & & & & & & & & & & & & & & & & & & & & & & & \\
\hline 105 & - & 174, & 1 & & 3 & $106-108$ & & & & & & & & & & & & & & & & & & & & & & & & & & & & & & & \\
\hline 174,5 & - & 184 & 1 & & 3 & $59-60$ & & & & & & & & & & & & & & & & & & & & & & & & & & & & & & & \\
\hline 184 & - & 193,5 & 1 & & 3 & $84-86$ & & & & & & & & & & & & & & & & & & & & & & & & & & & & & & & \\
\hline & & & 1 & & & CC & & & & & & & & & & & & & & & & & & & & & & & & & & & & & & & \\
\hline 193,5 & & 203 & 1 & & 3 & $88-90$ & & & & & & & & & & & & & & & & & & & & & & & & & & & & & & & \\
\hline & & & 1 & & & CC & & & & & & & & & & & & & & & & & & & & & & & & & & & & & & & \\
\hline 203 & - & 212,5 & 1 & & 3 & $87-89$ & & & & & & & & & & & & & & & & & & & & & & & & & & & & & & & \\
\hline 212,5 & & 222 & 1 & & 1 & $81-83$ & & & & & & & & & & & & & & & & & & & & & & & & & & & & & & & \\
\hline & & & 1 & & & $\mathrm{CC}$ & & & & & & & & & & & & & & & & & & & & & & & & & & & & & & & \\
\hline 222 & & 231,5 & 1 & & 2 & $61-63$ & & & & & & & & & & & & & & & & & & & & & & & & & & & & & & & \\
\hline & & & \begin{tabular}{|l}
1 \\
\end{tabular} & & & CC & & & & & & & & & & & & & & & & & & & & & & & & & & & & & & & \\
\hline 231,5 & & 241 & & & 1 & $123-125$ & & & & & & & & & & & & & & & & & & & & & & & & & & & & & & & \\
\hline & & & 1 & & & $C C$ & & & & & & & & & & & & & & & & & & & & & & & & & & & & & & & \\
\hline 241 & & 250, & 1 & & 1 & $127-129$ & & & & & & & & & & & & & & & & & & & & & & & & & & & & & & & \\
\hline & & & 1 & & & CC & & & & & & & & & & & & & & & & & & & & & & & & & & & & & & & \\
\hline 260 & & 269,5 & 1 & & 4 & $107-109$ & & & & & & & & & & & & & & & & & & & & & & & & & & & & & & & \\
\hline & & & 1 & & & $\mathrm{CC}$ & & & & & & & & & & & & & & & & & & & & & & & & & & & & & & & \\
\hline 279 & & 288,5 & 2 & & 1 & $102-104$ & & & & & & & & & & & & & & & & & & & & & & & & & & & & & & & \\
\hline 298 & & 307,5 & 2 & & 3 & $82-84$ & & & & & & & & & & & & & & & & & & & & & & & & & & & & & & & \\
\hline & & & 2 & & & $C C$ & & & & & & & & & & & & & & & & & & & & & & & & & & & & & & & \\
\hline 317 & & 326,5 & 2 & & 5 & $61-63$ & & & & & & & & & & & & & & & & & & & & & & & & & & & & & & & \\
\hline 336 & & 345,9 & 2 & & 3 & $93-95$ & & & & & & & & & & & & & & & & & & & & & & & & & & & & & & & \\
\hline & & & 2 & & & $C C$ & & & & & & & & & & & & & & & & & & & & & & & & & & & & & & & \\
\hline 355 & & 364, & 2 & & 2 & $12-14$ & & & & & & & & & & & & & & & & & & & & & & & & & & & & & & & \\
\hline & & & 2 & & & CC & & & & & & & & & & & & & & & & & & & & & & & & & & & & & & & \\
\hline 374 & & 383,5 & 2 & & 1 & $86-88$ & & & & & & & & & & & & & & & & & & & & & & & & & & & & & & & \\
\hline & & & 2 & & & CC & & & & & & & & & & & & & & & & & & & & & & & & & & & & & & & \\
\hline
\end{tabular}


TABLE 2 - Continued

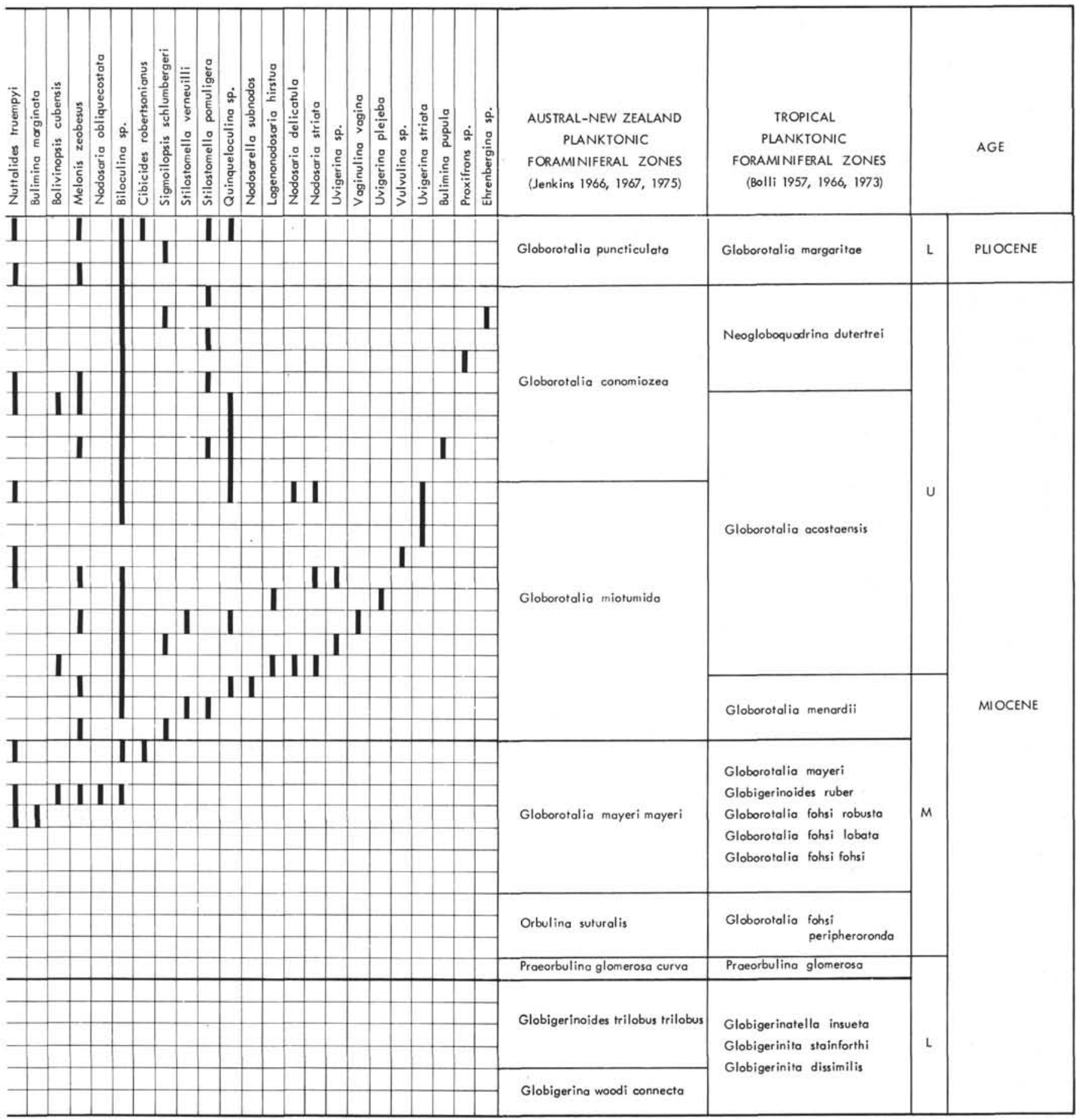


TABLE 2

Distribution of Pleistocene to Lower Miocene Benthic Foraminifers at Site 362

\begin{tabular}{|c|c|c|c|c|c|c|c|c|c|c|c|c|c|c|c|c|c|c|c|c|c|c|c|c|c|c|c|c|c|c|c|c|c|c|c|c|c|c|}
\hline & & & & & & & & & & & & & & & & & & & & & & & & & & & & & & & & & & & & & & \\
\hline 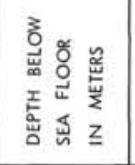 & 峞 & 吕 & $\begin{array}{l}\widehat{E} \\
\overrightarrow{\underline{z}} \\
\underline{\underline{z}} \\
\underline{\underline{z}}\end{array}$ & 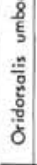 & 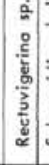 & 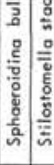 & 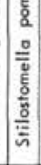 & 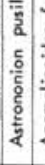 & 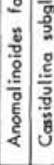 & 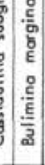 & 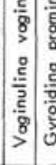 & . & $\begin{array}{l}0 \\
0 \\
0 \\
\frac{2}{2} \\
\frac{2}{8} \\
8\end{array}$ & 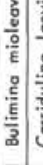 & 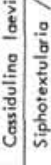 & 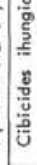 & 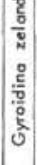 & 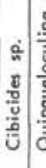 & 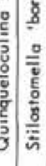 & 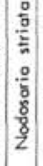 & 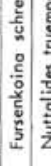 & 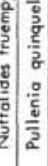 & 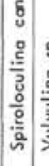 & 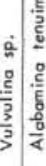 & 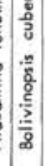 & 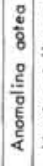 & & 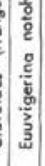 & 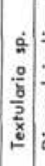 & 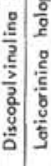 & 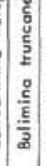 & 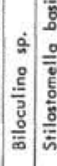 & 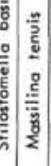 & 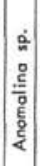 & & 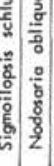 & & 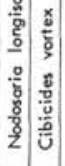 \\
\hline $36-45,5$ & 1 & 1 & $58-60$ & & & & & & & & & & & & & & & & & & & & & & & & & & & & & & & & & & & \\
\hline $45,5-55$ & 2 & 3 & $58-60$ & & & & & & & & & & & & & & & & & & & & & & & & & & & & & & & & & & & \\
\hline $55-44$ & 3 & 3 & $58-\infty 0$ & & & & & & & & & & & & & & & & & & & & & & & & & & & & & & & & & & & \\
\hline $64,5-74$ & 4 & 3 & \begin{tabular}{|c}
$58-60$ \\
$C C$
\end{tabular} & & & & & & & & & & & & & & & & & & & $\mathbf{I}$ & & & & & & I & & & & & & I & & & & \\
\hline $74-83$ & 5 & & $c c$ & & & & & & & & & & & & & & & & & & & & & & & & & . & & & & & & & & & & \\
\hline $83,5-93$ & 6 & 3 & $58-60$ & & & & & & & & & & & & & & & & & & & & & & & & & 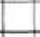 & & & & & & 1 & & & & \\
\hline $93-102,5$ & 7 & & $C C$ & & & & & & & & & & & & & & & & & & & & & & & & & & & & & & & & & & & \\
\hline $102,5-112$ & 8 & & $C C$ & & & & & & & & & & & & & & & & & & & & & & & & & & & & & & & & & & & \\
\hline $112-121,5$ & 9 & 3 & $58-60$ & & & & & & & & & & & & & & & & & & & & & & & & & & & & & & & & & & & \\
\hline $121,5-131$ & 10 & 3 & $58-60$ & & & & & & & & & & & & & & & & & & & & & & & & & & & & & & & & & & & \\
\hline $131-140,5$ & 11 & 1 & \begin{tabular}{|cc}
$58-60$ \\
$C C$
\end{tabular} & & & & & & & & & & & & & & & & & & & & & & & & & & & & & & I & 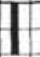 & & & & \\
\hline $140,5-150$ & 12 & & $\mathrm{CC}$ & & & & & & & & & & & & & & & & & & & 1 & & & & & & & I & & & & & & & & & \\
\hline $150-159,5$ & 13 & 3 & $\frac{58-60}{C C}$ & & & & & & & & & & & & & & & & & & & & & & & & & & & & & & 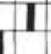 & & & & & \\
\hline $159,5-169$ & 14 & & $\mathrm{CC}$ & & & & & & & & & & & & & & & & & & & 1 & & & & & & & & & & & & & & & & \\
\hline $197,5-207$ & 16 & 4 & $58-60$ & & & & & & & & & & & & & & & & & & & & & & & & & & & & & & & & & & & \\
\hline $216,5-226$ & 17 & 4 & $58-60$ & & & & & & & & & & & & & & & & & & & & & & & & & & & & & & & & & & & \\
\hline $235,5-245$ & 18 & 3 & $58-60$ & & & & & & & & & & & & & & & & & & & & & & & & & & & & & & & & & & & \\
\hline $254,5-264$ & 19 & 3 & $\frac{58-60}{\mathrm{CC}}$ & & & & & & & & & & & & & & & & & & & & & & & & & & & & & & & & & & & \\
\hline $273,5-283$ & 20 & & CC & & & & & & & & & & & & & & & & & & & & & & & & & & & & & & & & & & & \\
\hline $292,5-302$ & 21 & 3 & $58-60$ & & & & & & & & & & & & & & & & & & & & & & & & & & & & & & & & & & & \\
\hline $311,5-321$ & 22 & & $\mathrm{CC}$ & & & & & & & & & & & & & & & & & & & & & & & & & & & & & & $I$ & & & & & \\
\hline $330,5-340$ & 23 & 3 & $58-60$ & & & & & & & & & & & & & & & & & & & & & & & & & & & & & & & & & & & \\
\hline $349,5-359$ & 24 & 3 & $58-60$ & & & & & & & & & & & & & & & & & & & & & & & & & & & & & & & & & & & \\
\hline $368,5-378$ & 25 & 3 & $58-60$ & & & & & & & & & & & & & & & & & & & & & & & & & & & & & & 1 & & & & & \\
\hline $387,5-397$ & 26 & 3 & $58-60$ & & & & & & & & & & & & I & & & & & & & & & & & & & & & & & & & & & & & \\
\hline $406,5-416$ & 27 & 3 & $58-60$ & & & & & & & & & & & & & & & & & & & & & & & & & & & & & & & & & & & \\
\hline $425,5-435$ & 28 & 3 & $58-60$ & & & & & & & & & & & & & & & & & & & & & & & & & & & & & & 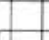 & & & & 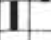 & \\
\hline $444,5-454$ & 29 & 3 & $\begin{array}{l}58-60 \\
C C\end{array}$ & & & & & & & & & & & & & & & & & & & & & & & & & & & & & & & & & & I & \\
\hline $463,5-473$ & 30 & 3 & $\frac{58-60}{C C}$ & & & & & & & & & I & & & & & & & & & & & & & & & & & & & & & & & & & & \\
\hline $482,5-492$ & 31 & $\frac{1}{3}$ & $\frac{58-60}{58-60}$ & & & & & & & & & & & & & & & & & & & & & 【 & & & & & & & & I & & & & & & \\
\hline & - & & CC & & & & & & I & & & I & & & I & & & & & & & & & & & & & & & & 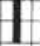 & $I$ & & & & & & \\
\hline $501,5-511$ & 32 & 3 & $\frac{58-60}{\mathrm{CC}}$ & & & & & & & & & & & & & & & & & & & & & & & 8 & & & & & & & & & & & & \\
\hline $520,5-530$ & 33 & $\frac{4}{5}$ & \begin{tabular}{|l|}
$58-60$ \\
$58-60$
\end{tabular} & & & & & & & & & & & & I & & & & & & & & & & & & & & & & & & & & & & $I$ & I \\
\hline & & 6 & $\frac{38-60}{58-60}$ & & & & & & & & & & & & & & & & & & & & & & & I & & & & & & 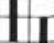 & & & & & I & \\
\hline $549-559,5$ & 34 & & CC & & & & & & & & & & & & & & & & & & & & & & & & & & & & & & & & & & & \\
\hline $577,5-587$ & 35 & $\frac{3}{5}$ & $\frac{58-60}{58-60}$ & & & & & & & & & & & & & & & & & & & 1 & & & & & & & & & & t & & & 1 & & & \\
\hline & & & $\frac{58-60}{C C}$ & & & & & & & & & & & & 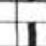 & & & & & & & & & & & & & & & & & & & & & & & \\
\hline $596,5-606$ & 36 & 3 & $\frac{87-89}{58-60}$ & & & & & & & & & & & & & & & & & & I & & & & & & & & & & & & & & & & & \\
\hline $615,5-625$ & 37 & 2 & & & & & & & & & & & & & & & & & & & & I & & & & & & & & & & & & & & & & \\
\hline & & 4 & 58 & & & & & & & & & & & & 1 & & & & 1 & & 1 & & & & & & & & & & & & & & & & & \\
\hline $644-653,5$ & 38 & 1 & $58-60$ & & & & & & & & & & & & & & & & & & & & & & & & & & & & & & & & & & & \\
\hline
\end{tabular}


TABLE 2 - Continued

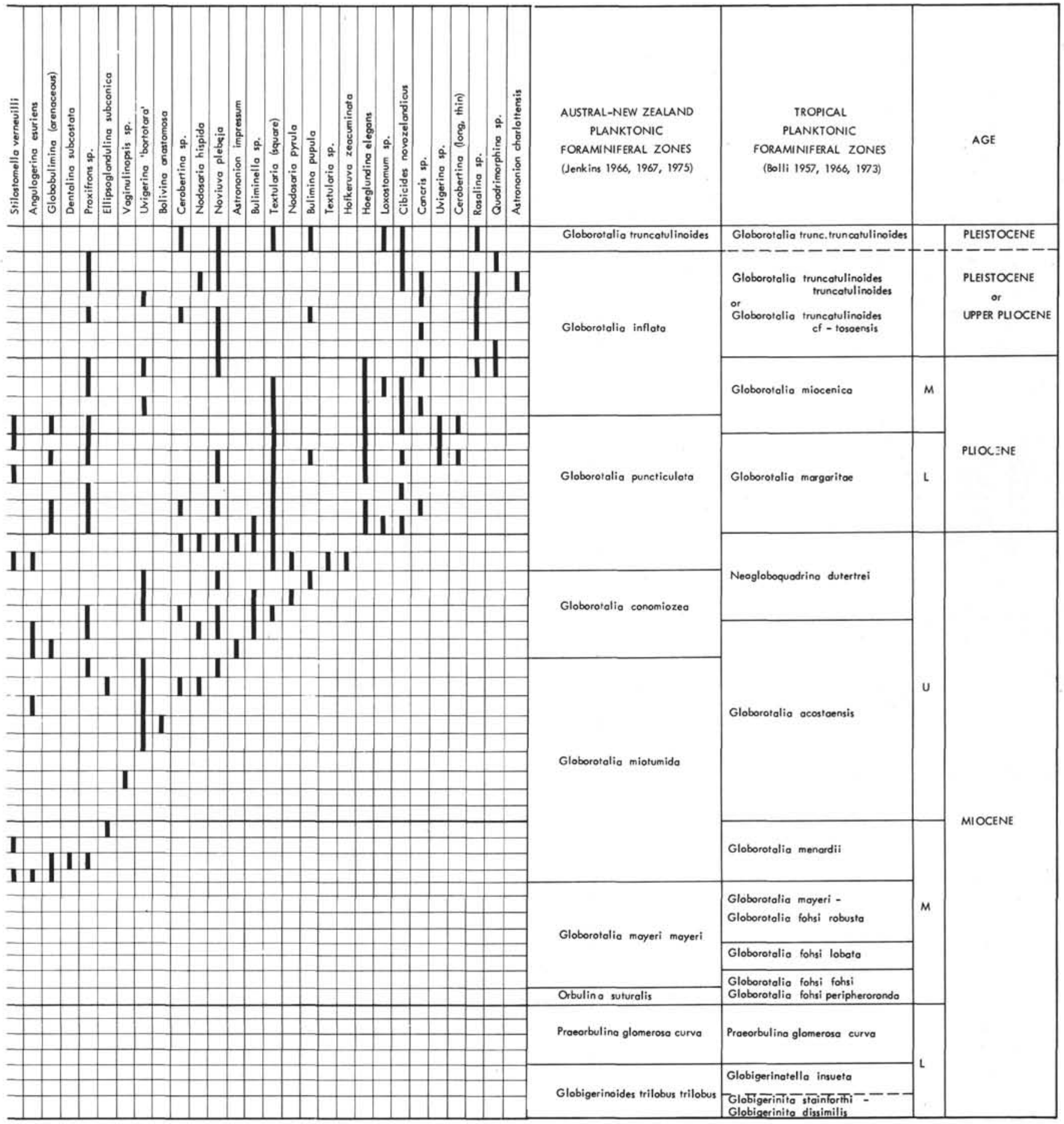




\section{PLATE 1}

Figure 1 Bolivinopsis cubensis (Cushman and Bermudez). $\times 41$.

Sample 362-19, CC. Globorotalia conomiozea Zone; upper Miocene.

Figure 2 Massilina tenuis (Czjzek). $\times 47$.

Sample 362-19, CC. Globorotalia conomiozea Zone; upper Miocene.

Figure $3 \quad$ Vaginulina vagina $($ Stache) $\times 23$.

Sample 362-19, CC. Globorotalia conomiozea Zone; upper Miocene.

Figure $4 \quad$ Vaginulinopsis spinulosa (Stache). $\times 16$.

Sample 360-23, CC. Globigerinoides trilobus trilobus Zone; lower Pliocene.

Figures 5,6 Plectofrondicularia whaingaroica (Stache). $\times 29$.

Sample 362-11, CC. Globorotalia puncticulata Zone; lower Pliocene.

Figure $7 \quad$ Stilostomella basicarinata Hornibrook. $\times 29$.

Sample 362-14, CC. Globorotalia puncticulata Zone; lower Pliocene.

Figure $8 \quad$ Stilostomella pomuligera $($ Stache). $\times 29$. Sample 362-8, CC. Globorotalia inflata Zone; middle Pliocene.

Figure $9 \quad$ Stilostomella stachei (Chapman). $\times 29$.

Sample 362-20, CC. Globorotalia conomiozea Zone; upper Miocene.

Figure $10 \quad$ Bulimina truncanella Finlay. $\times 70$.

Sample 362-20, CC. Globorotalia conomiozea Zone; upper Miocene.

Figure $11 \quad$ Angulogerina esuriens Hornibrook. $\times 99$.

Sample $362-17-4, \quad 58-60 \mathrm{~cm}$. Globorotalia puncticulata Zone; upper Miocene.

Figure $12 \quad$ Cassidulina subglobosa Brady. $\times 68$.

Sample 362-20, CC. Globorotalia conomiozea Zone; upper Miocene.

Figures 13,14 Pullenia bulloides (d'Orbigny). $\times 59$.

Sample 362-8, CC. Globorotalia inflata Zone; middle Pliocene.

Figures 15, 16 Pullenia quinqueloba (Reuss). $\times 59$.

Sample 362-4, CC. Globorotalia inflata Zone; Pleistocene to upper Pliocene.

Figure 17 Alabamina tenuimarginata (Chapman, Parr and Collins). $\times 29$.

Sample 362-20, CC. Globorotalia conomiozea Zone; upper Miocene.

Figures 18-20 Gyroidina zelandicus (Finlay). $\times 29$.

Sample 362-19, CC. Globorotalia conomiozea Zone; upper Miocene.

Figures 21-23 Anomalina aotea Finlay. $\times 50$.

Sample 362-20, CC. Globorotalia conomiozea Zone; upper Miocene.

Figures 24, 25 Anomalinoides fasciatus (Stache). $\times 47$. Sample 362-8, CC. Globorotalia inflata Zone; middle Pliocene. 
PLATE 1

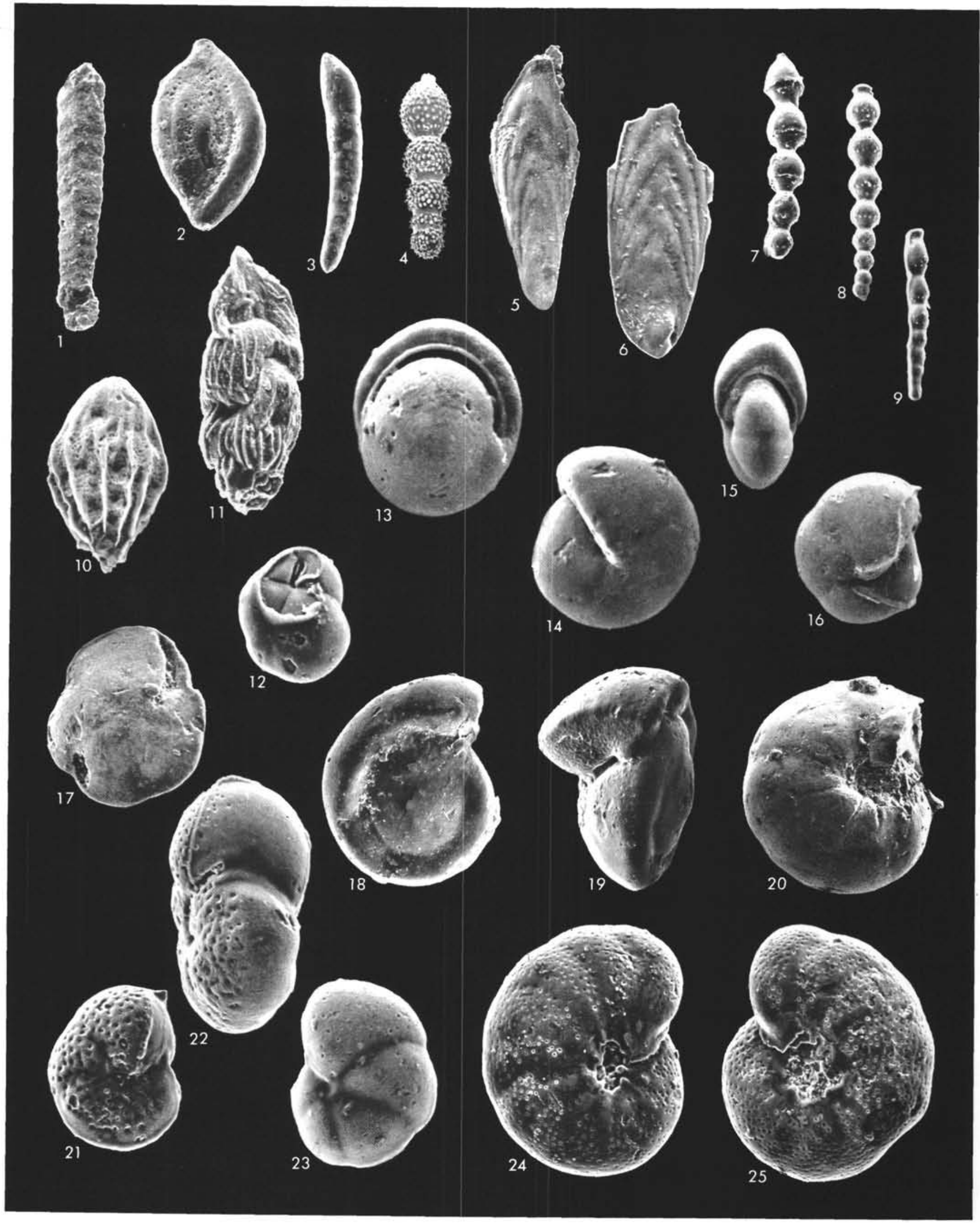

